\title{
PLANAR AND PROJECTIVE LINE GRAPHS OF COMAXIMAL GRAPHS OF LATTICES
}

Mojgan Afkhami, Kazem Khashyarmanesh and Atossa Parsapour

Received: 11 February 2014; Revised: 18 March 2014

Communicated by A. Çiğdem Özcan

ABstract. In this paper, we study all planar and projective line graphs associated to a comaximal graph of a finite lattice.

Mathematics Subject Classification (2010): 05C10, 06A07, 06B10

Keywords: Comaximal graph, lattice, line graph, planar graph, projective graph

\section{Introduction}

The concept of a comaximal graph of a commutative ring $R$ was first defined in [14]. In [14], Sharma and Bhatwadekar defined the comaximal graph of $R$, denoted by $\Gamma(R)$, with all elements of $R$ being the vertices, and two distinct vertices $a$ and $b$ are adjacent if and only if $R a+R b=R$. In [8] and [15], the authors considered a subgraph $\Gamma_{2}(R)$ of $\Gamma(R)$ consisting of non-unit elements of $R$, and studied several properties of the comaximal graph. Also the comaximal graph of a non-commutative ring was defined and studied in [16]. Recently, in [1], the comaximal graph of a lattice was defined and studied.

The comaximal graph of a lattice $L=(L, \wedge, \vee)$, denoted by $\Gamma(L)$, is an undirected graph with all elements of $L$ being the vertices, and two distinct vertices $a$ and $b$ are adjacent if and only if $a \vee b=1$.

One important theorem due to Whitney about the line graphs is that with one exceptional case, $\mathfrak{L}(G)=K_{3}$, the structure of any connected graph can be recovered from its line graphs, i.e., there is one-to-one correspondence between the class of connected graphs and the class of connected line graphs (see [17]). Hence in this paper we consider the line graph of the comaximal graph of a finite lattice.

In this work, we denote by $\mathfrak{L}\left(\Gamma_{2}(L)\right)$, the line graph associated to $\Gamma_{2}(L)$. In Section 2, we study the planarity of the line graph associated to comaximal graph of finite lattices. In Section 3, we characterize all finite lattice $L$ that, the line graph $\mathfrak{L}\left(\Gamma_{2}(L)\right)$ is projective. 
First we recall some definitions and notations on lattices and graphs. Recall that a lattice is a set $L=(L, \wedge, \vee)$ with two binary operations $\wedge$ and $\vee$, satisfying the following conditions: for all $a, b, c \in L$,

1. $a \wedge a=a, a \vee a=a$,

2. $a \wedge b=b \wedge a, a \vee b=b \vee a$,

3. $(a \wedge b) \wedge c=a \wedge(b \wedge c), a \vee(b \vee c)=(a \vee b) \vee c$, and

4. $a \vee(a \wedge b)=a \wedge(a \vee b)=a$.

Note that in every lattice the equality $a \wedge b=a$ always implies that $a \vee b=b$. Also, by [11, Theorem 2.1], one can define an order $\leqslant$ on $L$ as follows:

For any $a, b \in L$, we set $a \leqslant b$ if and only if $a \wedge b=a$. Then $(L, \leqslant)$ is an ordered set in which every pair of elements has a greatest lower bound (g.l.b.) and a least upper bound (l.u.b.). Conversely, let $P$ be an ordered set such that, for every pair $a, b \in P$, g.l.b.(a,b) and l.u.b.(a,b) belong to $P$. For each $a$ and $b$ in $P$, we define $a \wedge b:=$ g.l.b. $(a, b)$ and $a \vee b:=$ l.u.b. $(a, b)$. Then $(P, \wedge, \vee)$ is a lattice.

Recall that in a partially ordered set $(P, \leqslant)$, we say that $a$ covers $b$ or $b$ is covered by $a$, in notation $b \prec a$, if and only if $b<a$ and there is no element $p$ in $P$ such that $b<p<a$. An element $a$ in $L$ is called a co-atom if $a \prec 1$. We denote the sets of all co-atoms in a lattice $L$ by $C(L)$. Also, for an element $a \in L$, we have $[a]^{l}=\{b \in L \mid b \leq a\}$.

Now, we recall some definitions and notations on graphs. Let $G$ be a simple graph with vertex-set $V(G)$ and edge-set $E(G)$. In a graph $G$, for two distinct vertices $a$ and $b$ in $G$, the notation $a-b$ means that $a$ and $b$ are adjacent. Also, the degree of a vertex $x$, denoted by $\operatorname{deg}(x)$, is the number of edges incident to $x$, and an isolated vertex is a vertex with zero degree. A graph with no edges (but at least one vertex) is called an empty graph. The graph with no vertices and no edges is the null graph. For a positive integer $r$, an $r$-partite graph is one whose vertex-set can be partitioned into $r$ subsets, so that no edge has both ends in any one subset. A complete r-partite graph is one in which each vertex is joined to every vertex that is not in the same subset. For positive integers $m$ and $n$, the graph $K_{n}$ is a complete graph with $n$ vertices and the graph $K_{m, n}$ is a complete bipartite graph, with parts of sizes $m$ and $n$. A complete bipartite graph $K_{1, n}$ is called star (see [3] and [7]). A graph $G$ is said to be contracted to a graph $H$ if there exists a sequence of elementary contractions which transforms $G$ into $H$, where an elementary contraction consists of deletion of a vertex or an edge or the identification of two adjacent vertices. A subdivision of a graph is any graph that can be obtained from the original graph by replacing edges by paths. The line 
graph of a graph $G$ is the graph $\mathfrak{L}(G)$ with the edges of $G$ as its vertices, and two edges of $G$ are adjacent in $\mathfrak{L}(G)$ if and only if they are incident in $G$. In this work, we denote $w_{i, j}$ for the vertex $\left[v_{i}, v_{j}\right] \in \mathfrak{L}(G)$, where $v_{i}$ and $v_{j}$ are adjacent vertices in $G$.

Recall that a graph is said to be planar if it can be drawn in the plane, such that its edges intersect only at their ends. A remarkable characterization of the planar graphs was given by Kuratowski in 1930. Kuratowski's Theorem says that a graph is planar if and only if it contains no subdivision of $K_{5}$ or $K_{3,3}$ (cf. [3, p. 153]). In [13], the author characterized the planarity of a line graph $\mathfrak{L}(G)$ by using the planarity of $G$ and its vertex degrees.

Theorem 1.1. [13, Lemma 2.6] A non-empty graph $G$ has a planar line graph $\mathfrak{L}(G)$ if and only if

(i) $G$ is planar,

(ii) $\triangle(G) \leq 4$, and

(iii) if $\operatorname{deg}(v)=4$, then $v$ is a cut-vertex in the graph $G$.

By a surface, we mean a connected compact 2-dimensional real manifold without boundary, that is a connected topological space such that each point has a neighbourhood homeomorphic to an open disc. It is well-known that every compact surface is homeomorphic to a sphere, or to a connected sum of $g$ tori, or to a connected sum of $k$ projective planes (see [9, Theorem 5.1]). This number $k$ is called the crosscap number of the surface. Also, we denote the crosscap number of a graph $G$ by $\bar{\gamma}(G)$. One easy observation is that $\bar{\gamma}(H) \leq \bar{\gamma}(G)$, for any subgraph $H$ of $G$. The projective plane can be though of as a sphere with one crosscap. This means that the crosscap number of projective plane is 1 .

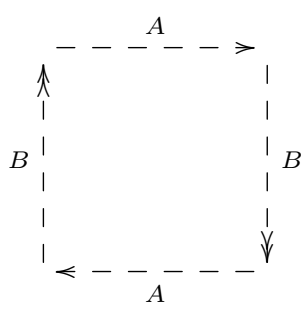

The canonical representation of a projective plane

A graph $G$ is embeddable in a surface $S$ if the vertices of $G$ are distinct points in $S$ and every edge of $G$ is a simple arc in $S$ connecting the two vertices which is joined in $G$. A projective graph is a graph that can be embedded in a projective 
plane. If $G$ can not be embedded in $S$, then $G$ has at least two edges intersecting at a point which is not a vertex of $G$. We say a graph $G$ is irreducible for a surface $S$ if $G$ does not embed in $S$, but any proper subgraph of $G$ embeds in $S$. The set of 103 irreducible graphs for the projective plane has been found by H. Glover, J.P. Huneke and C.S. Wang in [6], and D. Archdeacon in [2] proved that this list is complete. This list also has been checked by W. Myrvold and J. Roth in [10]. Hence a graph embeds in the projective plane if and only if it contains no subdivision of 103 graphs in [6]. Also, a complete graph $K_{n}$ is projective if $n=5$ or 6 , and the only projective complete bipartite graphs are $K_{3,3}$ and $K_{3,4}$ (see [4] or [12]). Note that a planar graph is not considered as a projective graph. In this paper, we assume that $L$ is a finite lattice. The comaximal graph of a lattice $L$, denoted by $\Gamma(L)$, is an undirected graph with all elements of $L$ being the vertices, and two distinct vertices $a$ and $b$ are adjacent if and only if $a \vee b=1$ (see [1]). It is easy to see that 1 is adjacent to all vertices, and so $\Gamma(L)$ is a refinement of a star graph with center 1. Thus we consider an induced subgraph of $\Gamma(L)$ with vertex set $L \backslash\{1\}$. Also the vertices in the set $J(L)$ are isolated vertices in this subgraph, since they are adjacent to 1 , where $J(L)$ is the set $\bigcap_{m \in C(L)} m$. Thus we denote the induced subgraph of $\Gamma(L)$ with vertex set $L \backslash(J(L) \cup\{1\})$, by $\Gamma_{2}(L)$, and in the rest of the paper we deal with the graph $\Gamma_{2}(L)$.

\section{On the planarity of $\mathfrak{L}\left(\Gamma_{2}(L)\right)$}

In this section, we explore the planarity of the line graph associated to the graph $\Gamma_{2}(L)$, where $L$ is a finite lattice. In fact, we characterize all finite lattices in terms of the size of their co-atoms. If $|C(L)|=1$, then $\Gamma_{2}(L)$ is an empty graph, and hence $\mathfrak{L}\left(\Gamma_{2}(L)\right)$ is a null graph. We begin this section with the following notation, which is needed in the rest of the paper.

Notation 2.1. Suppose that $|C(L)|=t$, where $t>1$. To simplify notation, we denote the set $[m]^{l}$, where $m \in C(L)$, by $\mathfrak{m}$. We set $S_{j}:=\mathfrak{m}_{j} \backslash \bigcup_{i \notin\{j\}} \mathfrak{m}_{i}$, where $1 \leq i, j \leq t$. Also, $S_{j_{1} j_{2} \ldots j_{k}}:=\left(\mathfrak{m}_{j_{1}} \cap \mathfrak{m}_{j_{2}} \cap \cdots \cap \mathfrak{m}_{j_{k}}\right) \backslash \bigcup_{i \notin\left\{j_{1}, j_{2}, \ldots, j_{k}\right\}} \mathfrak{m}_{i}$, where $1 \leq j_{1}<j_{2}<\cdots<j_{k} \leq t$. Note that each element in $S_{i}$ is adjacent to all elements of $S_{j}$, for $i \neq j$, and also it is adjacent to all elements of $S_{j_{1} j_{2} \cdots j_{k}}$, where $j_{1}, \ldots, j_{k} \notin\{i\}$.

Now, we state the following lemma.

Lemma 2.2. If $\mathfrak{L}\left(\Gamma_{2}(L)\right)$ is planar, then the size of $C(L)$ is at most four. 
Proof. Assume to the contrary that $|C(L)| \geq 5$. Then the graph $\Gamma_{2}(L)$ contains a copy of $K_{5}$ with vertices $v_{1} \in S_{1}, v_{2} \in S_{2}, v_{3} \in S_{3}, v_{4} \in S_{4}$ and $v_{5} \in S_{5}$. So the graph $\mathfrak{L}\left(\Gamma_{2}(L)\right)$ contains a subdivision of $K_{3,3}$ (see Figure 1).

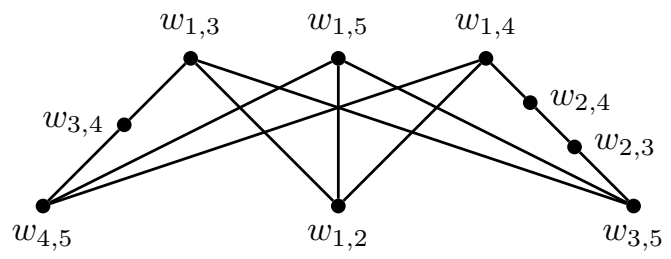

FIGURE 1

Therefore, it is not a planar graph, which is a contradiction.

By Lemma 2.2, it is sufficient for us to investigate the planarity of the graph $\mathfrak{L}\left(\Gamma_{2}(L)\right)$ in the cases in which the size of $C(L)$ is 2,3 and 4 .

First we state the necessary and sufficient condition for the planarity of the graph $\mathfrak{L}\left(\Gamma_{2}(L)\right)$, when $|C(L)|=2$.

Theorem 2.3. Suppose that $|C(L)|=2$. Then $\mathfrak{L}\left(\Gamma_{2}(L)\right)$ is a planar graph if and only if $\left|\bigcup_{j=1}^{2} S_{j}\right| \leq 5$.

Proof. First, assume that $\mathfrak{L}\left(\Gamma_{2}(L)\right)$ is planar and assume on the contrary that $\left|\bigcup_{j=1}^{2} S_{j}\right| \geq 6$. We know that as $|C(L)|=2$, the graph $\Gamma_{2}(L)$ is a complete bipartite graph. If $\Gamma_{2}(L)$ is a star graph, then the graph $\mathfrak{L}\left(\Gamma_{2}(L)\right)$ contains a subgraph isomorphic to $K_{5}$, which is not planar. Otherwise, $\Gamma_{2}(L)$ is not a star graph. Then it contains a subgraph isomorphic to $K_{2,4}$ or $K_{3,3}$. In these two cases, $\mathfrak{L}\left(\Gamma_{2}(L)\right)$ contains a subdivision of $K_{3,3}$. Hence $\mathfrak{L}\left(\Gamma_{2}(L)\right)$ is not planar, which is a contradiction.

Conversely, suppose that $\left|\bigcup_{j=1}^{2} S_{j}\right| \leq 5$. If $\left|\bigcup_{j=1}^{2} S_{j}\right|=2$, then $\mathfrak{L}\left(\Gamma_{2}(L)\right)$ is isomorphic to $\mathfrak{L}\left(K_{2}\right)$, which is an empty graph with one vertex. Also if $\left|\bigcup_{j=1}^{2} S_{j}\right|=$ 3 , then $\mathfrak{L}\left(\Gamma_{2}(L)\right) \cong \mathfrak{L}\left(K_{1,2}\right) \cong K_{2}$. In addition, if $\left|\bigcup_{j=1}^{2} S_{j}\right|=4$, then $\Gamma_{2}(L)$ is isomorphic to $K_{1,3}$ or $K_{2,2}$. Hence $\mathfrak{L}\left(\Gamma_{2}(L)\right)$ is isomorphic to $K_{3}$ and $K_{2,2}$, respectively. Finally, assume that $\left|\bigcup_{j=1}^{2} S_{j}\right|=5$. If $\Gamma_{2}(L)$ is a star graph, then $\mathfrak{L}\left(\Gamma_{2}(L)\right) \cong K_{4}$. Otherwise, the graph $\Gamma_{2}(L)$ is isomorphic to $K_{2,3}$ with vertices $v_{1}, v_{2}, v_{3} \in S_{1}$ and $v_{4}, v_{5} \in S_{2}$. In this case, the graph $\mathfrak{L}\left(\Gamma_{2}(L)\right)$ is pictured in Figure 2 .

In all of the above situations, $\mathfrak{L}\left(\Gamma_{2}(L)\right)$ is a planar graph. 


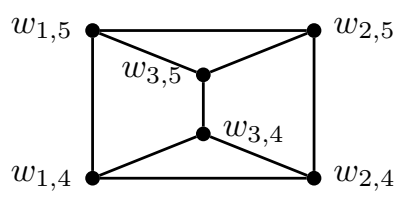

FIGURE 2

Now, we investigate the planarity of $\mathfrak{L}\left(\Gamma_{2}(L)\right)$, when $|C(L)|=3$. Let $\left|\bigcup_{j=1}^{3} S_{j}\right| \geq$ 5. It is easy to see that $\Gamma_{2}(L)$ contains a subgraph isomorphic to a complete 3partite graph $K_{3,1,1}$ or $K_{2,2,1}$. Therefore the graph $\mathfrak{L}\left(\Gamma_{2}(L)\right)$ contains a subdivision of $K_{3,3}$ and a subdivision of $K_{5}$, respectively. Hence it is not planar, and so we have the following lemma.

Lemma 2.4. If $\mathfrak{L}\left(\Gamma_{2}(L)\right)$ is planar, then $\left|\bigcup_{j=1}^{3} S_{j}\right| \leq 4$.

Theorem 2.5. Suppose that $|C(L)|=3$. Then $\mathfrak{L}\left(\Gamma_{2}(L)\right)$ is a planar graph if and only if one of the following conditions hold:

(i) $\left|\bigcup_{j=1}^{3} S_{j}\right|=3$ and $\left|S_{i j}\right| \leq 2$, for $1 \leq i, j \leq 3$.

(ii) $\left|\bigcup_{j=1}^{3} S_{j}\right|=4$ and $\left|S_{i j}\right| \leq 1$, for $1 \leq i, j \leq 3$.

Proof. First, assume that one of the conditions (i) or (ii) holds. Suppose that $\left|\bigcup_{j=1}^{3} S_{j}\right|=3$ and $\left|S_{23}\right|=\left|S_{12}\right|=\left|S_{13}\right|=2$. The graph $\Gamma_{2}(L)$ with vertices $v_{1} \in S_{1}, v_{2} \in S_{2}, v_{3} \in S_{3}, v_{4}, v_{5} \in S_{23}, v_{6}, v_{7} \in S_{12}$ and $v_{8}, v_{9} \in S_{13}$ is pictured in Figure 3 .

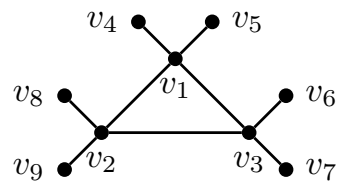

FiguRE 3

Hence the graph $\mathfrak{L}\left(\Gamma_{2}(L)\right)$ is pictured in Figure 4, which is planar.

Now, suppose that $\left|\bigcup_{j=1}^{3} S_{j}\right|=4,\left|S_{1}\right|=2$ and $\left|S_{23}\right|=\left|S_{13}\right|=\left|S_{12}\right|=1$. The graph $\Gamma_{2}(L)$ with vertices $v_{1}, v_{2} \in S_{1}, v_{3} \in S_{2}, v_{4} \in S_{3}, v_{5} \in S_{23}, v_{6} \in S_{13}$, $v_{7} \in S_{12}$ is pictured in Figure 5 and $\mathfrak{L}\left(\Gamma_{2}(L)\right)$ is pictured in Figure 6, which is a planar graph.

Conversely, suppose that $\mathfrak{L}\left(\Gamma_{2}(L)\right)$ is a planar graph. By Lemma 2.4, $\left|\bigcup_{j=1}^{3} S_{j}\right| \leq$ 4. Hence we have the following cases. 


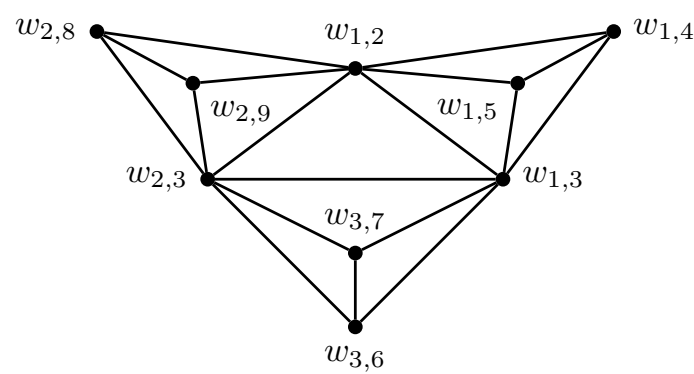

Figure 4

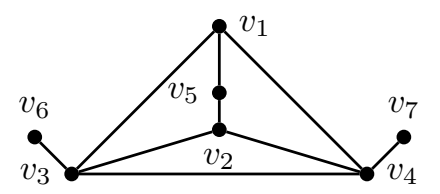

Figure 5

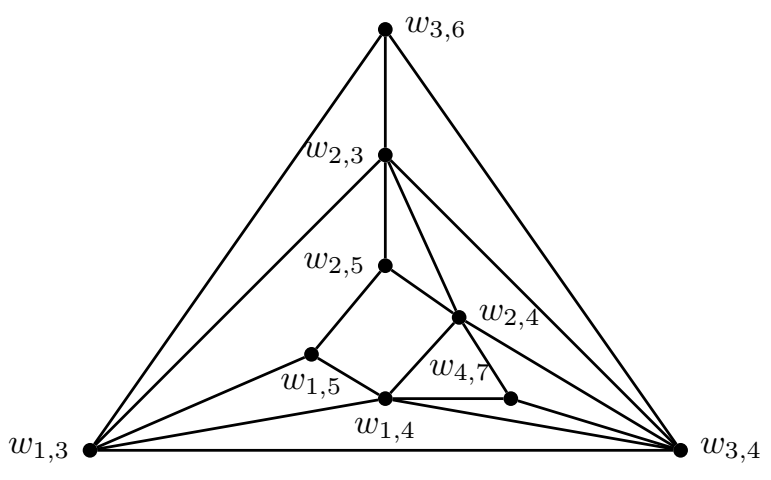

Figure 6

Case 1. $\left|\bigcup_{j=1}^{3} S_{j}\right|=3$. If $S_{12}, S_{13}$ or $S_{23}$ has at least three elements, then there exists at least a vertex of degree five in the graph $\Gamma_{2}(L)$. Hence the graph $\mathfrak{L}\left(\Gamma_{2}(L)\right)$ contains a subgraph isomorphic to $K_{5}$, and so it is not planar, which is a contradiction.

Case 2. $\left|\bigcup_{j=1}^{3} S_{j}\right|=4$. Without loss of generality, we may assume that $\left|S_{1}\right|=2$. If $S_{12}$ or $S_{13}$ has at least two elements, then there exists at least a vertex of degree five in the graph $\Gamma_{2}(L)$. Hence the graph $\mathfrak{L}\left(\Gamma_{2}(L)\right)$ contains a copy of $K_{5}$, and so it is not planar, which is a contradiction. In addition, if $S_{23}$ has at least two elements, then $\Gamma_{2}(L)$ contains a subgraph isomorphic to $K_{2,4}$. Therefore $\Gamma_{2}(L)$ has a vertex 
of degree four which is not a cut-vertex. By Theorem 1.1, $\mathfrak{L}\left(\Gamma_{2}(L)\right)$ is not a planar graph, which is a contradiction.

Now, we study the planarity of $\mathfrak{L}\left(\Gamma_{2}(L)\right)$, when $|C(L)|=4$.

Lemma 2.6. If $\mathfrak{L}\left(\Gamma_{2}(L)\right)$ is planar, then $\left|\bigcup_{j=1}^{4} S_{j}\right|=4$.

Proof. Suppose on the contrary that $\left|\bigcup_{j=1}^{4} S_{j}\right| \geq 5$. Then the graph $\Gamma_{2}(L)$ has a vertex of degree four which is not a cut-vertex. Hence, by Theorem 1.1, $\mathfrak{L}\left(\Gamma_{2}(L)\right)$ is not a planar graph, which is a contradiction.

Theorem 2.7. Suppose that $|C(L)|=4$. Then $\mathfrak{L}\left(\Gamma_{2}(L)\right)$ is a planar graph if and only if $S_{i j}=\varnothing$ and $\left|S_{i j k}\right| \leq 1$, for all $i, j, k \in\{1,2,3,4\}$.

Proof. First, assume that the graph $\mathfrak{L}\left(\Gamma_{2}(L)\right)$ is planar. By Lemma 2.6, we have $\left|\bigcup_{j=1}^{4} S_{j}\right|=4$. If there exists at least one element in $S_{i j}$, for $i, j \in\{1,2,3,4\}$, then one can easily check that the graph $\mathfrak{L}\left(\Gamma_{2}(L)\right)$ contains a subdivision of $K_{3,3}$, which is not planar. Also if one of the sets $S_{i j k}$ has at least two elements, for $i, j, k \in\{1,2,3,4\}$, then the graph $\Gamma_{2}(L)$ has a vertex of degree five. Hence the graph $\mathfrak{L}\left(\Gamma_{2}(L)\right)$ contains a copy of $K_{5}$, which is impossible.

Conversely, suppose that $S_{12}=S_{13}=S_{23}=\varnothing$ and $\left|S_{123}\right|=\left|S_{124}\right|=\left|S_{134}\right|=$ $\left|S_{234}\right|=1$. The graph $\Gamma_{2}(L)$ with vertices $v_{1} \in S_{1}, v_{2} \in S_{2}, v_{3} \in S_{3}, v_{4} \in S_{4}$, $v_{5} \in S_{123}, v_{6} \in S_{124}, v_{7} \in S_{134}$ and $v_{8} \in S_{234}$ is pictured in Figure 7 . Hence

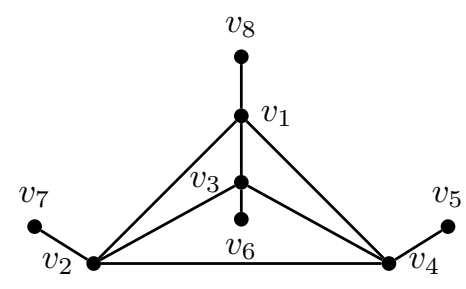

FiguRe 7

$\mathfrak{L}\left(\Gamma_{2}(L)\right)$ is pictured in Figure 8, which is a planar graph. Therefore, in the case that $S_{i j}=\varnothing$ and $\left|S_{i j k}\right| \leq 1$, for all $i, j, k \in\{1,2,3,4\}$, we have $\mathfrak{L}\left(\Gamma_{2}(L)\right)$ is planar.

\section{On the projectivity of $\mathfrak{L}\left(\Gamma_{2}(L)\right)$}

In this section, we investigate the projectivity of the line graph of the graph $\Gamma_{2}(L)$, where $L$ is a finite lattice. We begin this section with the following lemma.

Lemma 3.1. If $\mathfrak{L}\left(\Gamma_{2}(L)\right)$ is projective, then the size of $C(L)$ is at most four. 


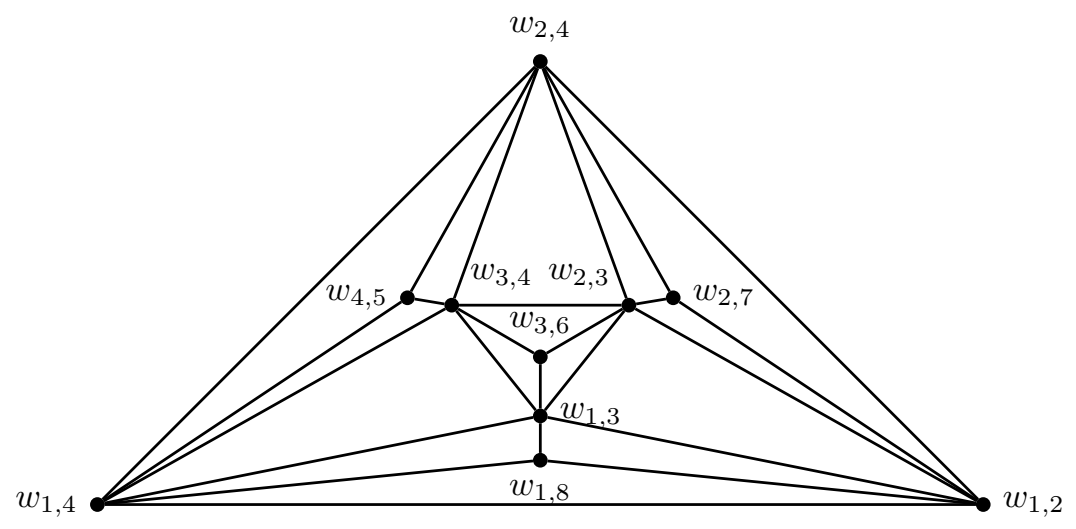

Figure 8

Proof. Assume to the contrary that $|C(L)| \geq 5$. Then the graph $\Gamma_{2}(L)$ contains a copy of $K_{5}$ with vertices $v_{1} \in S_{1}, v_{2} \in S_{2}, v_{3} \in S_{3}, v_{4} \in S_{4}$ and $v_{5} \in S_{5}$. So the contraction of the graph $\mathfrak{L}\left(\Gamma_{2}(L)\right)$ contains a copy of $E_{20}$, one of the listed graphs in [6] (see Figure 9). Therefore $\mathfrak{L}\left(\Gamma_{2}(L)\right)$ is not a projective graph, which is a contradiction.

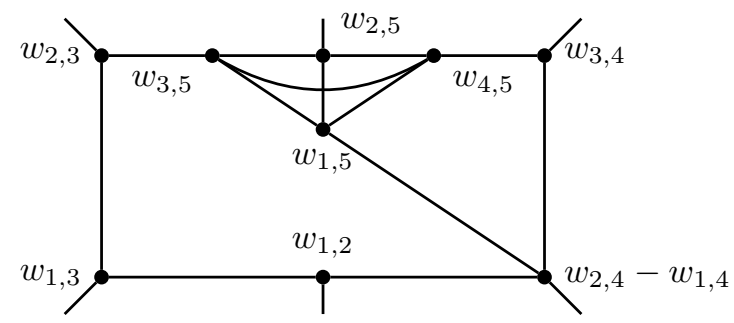

Figure 9

By Lemma 3.1, it is sufficient to investigate the projectivity of the graph $\mathfrak{L}\left(\Gamma_{2}(L)\right)$ in the cases that the size of $C(L)$ is 2,3 and 4 .

Theorem 3.2. Suppose that $|C(L)|=2$. Then $\mathfrak{L}\left(\Gamma_{2}(L)\right)$ is a projective graph if and only if one of the following conditions holds:

(i) $\left|\bigcup_{j=1}^{2} S_{j}\right|=6$ and $\left|S_{i}\right|=1$, for some unique $i \in\{1,2\}$ or $\left|S_{i}\right|=\left|S_{j}\right|=3$, for $i, j \in\{1,2\}$.

(ii) $\left|\bigcup_{j=1}^{2} S_{j}\right|=7$ and $\left|S_{i}\right|=1$, for some unique $i \in\{1,2\}$.

Proof. First, assume that the graph $\mathfrak{L}\left(\Gamma_{2}(L)\right)$ is projective and on the contrary, $\left|\bigcup_{j=1}^{2} S_{j}\right| \leq 5$. Then, by Theorem 2.3 , the graph $\mathfrak{L}\left(\Gamma_{2}(L)\right)$ is planar, which is not 
projective. Now, assume that $\left|\bigcup_{j=1}^{2} S_{j}\right|=6$ and $\Gamma_{2}(L) \cong K_{2,4}$. By $[5$, Example 2.14], $\bar{\gamma}\left(\mathfrak{L}\left(K_{2,4}\right)\right)=2$, and so the graph $\mathfrak{L}\left(\Gamma_{2}(L)\right)$ is not projective. Hence, if $\left|\bigcup_{j=1}^{2} S_{j}\right|=6$, then the statement (i) holds. Now, suppose that $\left|\bigcup_{j=1}^{2} S_{j}\right|=7$. If $\Gamma_{2}(L)$ is not a star graph, then it is isomorphic to $K_{2,5}$ or $K_{3,4}$. By [5, Corollary 2.11], $\bar{\gamma}\left(\mathfrak{L}\left(K_{2,5}\right)\right)=2$ and, by [5, Example 2.14], $\bar{\gamma}\left(\mathfrak{L}\left(K_{3,4}\right)\right)=2$. So if $\left|\bigcup_{j=1}^{2} S_{j}\right|=$ 7, then the statement (ii) holds. Finally, we may assume that $\left|\bigcup_{j=1}^{2} S_{j}\right| \geq 8$. If $\Gamma_{2}(L)$ is a star graph, then the graph $\mathfrak{L}\left(\Gamma_{2}(L)\right)$ contains a subgraph isomorphic to $K_{7}$, which is not projective. Otherwise, $\Gamma_{2}(L)$ is not a star graph. Then it contains a subgraph isomorphic to $K_{2,6}, K_{3,5}$ or $K_{4,4}$. In these cases, $\Gamma_{2}(L)$ contains a copy of $K_{2,4}$. Clearly, $\bar{\gamma}\left(\mathfrak{L}\left(\Gamma_{2}(L)\right)\right) \geq \bar{\gamma}\left(\mathfrak{L}\left(K_{2,4}\right)\right)$, and we have $\bar{\gamma}\left(\mathfrak{L}\left(K_{2,4}\right)\right)=2$. It means that the graph $\mathfrak{L}\left(\Gamma_{2}(L)\right)$ is not projective. Therefore, if $\mathfrak{L}\left(\Gamma_{2}(L)\right)$ is projective, then one of the conditions (i) or (ii) holds.

Conversely, suppose that $\left|\bigcup_{j=1}^{2} S_{j}\right|=6$, and the graph $\Gamma_{2}(L)$ is a star graph. Then $\mathfrak{L}\left(\Gamma_{2}(L)\right) \cong K_{5}$, and so it is a projective graph. Now, suppose that $\Gamma_{2}(L) \cong$ $K_{3,3}$. By [5, Example 2.12], $\bar{\gamma}\left(\mathfrak{L}\left(K_{3,3}\right)\right)=1$, and so the graph $\mathfrak{L}\left(\Gamma_{2}(L)\right)$ is projective. Finally, suppose that $\left|\bigcup_{j=1}^{2} S_{j}\right|=7$, and the graph $\Gamma_{2}(L)$ is a star graph. Then $\mathfrak{L}\left(\Gamma_{2}(L)\right) \cong K_{6}$, and so it is a projective graph.

Now, we investigate the projectivity of $\mathfrak{L}\left(\Gamma_{2}(L)\right)$, when $|C(L)|=3$.

Suppose that $\left|\bigcup_{j=1}^{3} S_{j}\right| \geq 6$. Then the graph $\Gamma_{2}(L)$ contains a subgraph isomorphic to $K_{4,1,1}, K_{3,2,1}$ or $K_{2,2,2}$. If $\Gamma_{2}(L)$ contains a subgraph isomorphic to $K_{4,1,1}$, then one can easily find a copy of $A_{1}$, one of the listed graphs in [6], in the graph $\mathfrak{L}\left(\Gamma_{2}(L)\right)$, which is not projective. Also if $\Gamma_{2}(L)$ contains a subgraph isomorphic to $K_{3,2,1}$, then one can easily find a copy of $E_{20}$, one of the listed graphs in [6], in the contraction of $\mathfrak{L}\left(\Gamma_{2}(L)\right)$, which is not projective. Now, if $\Gamma_{2}(L)$ contains a subgraph isomorphic to $K_{2,2,2}$, then the contraction of $\mathfrak{L}\left(\Gamma_{2}(L)\right)$ contains a copy of $E_{3}$, one of the listed graphs in [6], which is not projective. Therefore $\mathfrak{L}\left(\Gamma_{2}(L)\right)$ is not a projective graph.

As a consequence of the above discussion, we state the following lemma.

Lemma 3.3. If $\mathfrak{L}\left(\Gamma_{2}(L)\right)$ is projective, then $\left|\bigcup_{j=1}^{3} S_{j}\right| \leq 5$.

Theorem 3.4. Suppose that $|C(L)|=3$. Then $\mathfrak{L}\left(\Gamma_{2}(L)\right)$ is a projective graph if and only if one of the following conditions holds:

(i) $\left|\bigcup_{j=1}^{3} S_{j}\right|=3$, there exist unique $i$ and $j$, with $1 \leq i, j \leq 3$, such that $3 \leq$ $\left|S_{i j}\right| \leq 4$ and $\left|S_{k k^{\prime}}\right| \leq 2$, for $k \in\{i, j\}$ and $\left\{k^{\prime}\right\}=\{1,2,3\} \backslash\{i, j\}$.

(ii) $\left|\bigcup_{j=1}^{3} S_{j}\right|=4$, there exists unique $i$, with $1 \leq i \leq 3$, such that $\left|S_{i}\right|=2$, and for $\{j, k\}=\{1,2,3\} \backslash\{i\}$, if $2 \leq\left|S_{i j}\right| \leq 3$, then $\left|S_{i k}\right| \leq 1$ and $S_{j k}=\varnothing$. 
(iii) $\left|\bigcup_{j=1}^{3} S_{j}\right|=5$,

(a) there exists unique $i$, with $1 \leq i \leq 3$, such that $\left|S_{i}\right|=3$, and for all $1 \leq j, k \leq 3, S_{j k}=\varnothing$.

(b) there exists unique $i$, with $1 \leq i \leq 3$, such that $\left|S_{i}\right|=1$, and for $\{j, k\}=$ $\{1,2,3\} \backslash\{i\},\left|S_{j k}\right| \leq 1$ and $S_{i j}=S_{i k}=\varnothing$.

Proof. First we assume that $\mathfrak{L}\left(\Gamma_{2}(L)\right)$ is a projective graph. By Lemma 3.3, $\left|\bigcup_{j=1}^{3} S_{j}\right| \leq 5$. We have the following cases.

Case 1. $\left|\bigcup_{j=1}^{3} S_{j}\right|=3$. In this case, if $\left|S_{i j}\right| \leq 2$, for all $i, j \in\{1,2,3\}$, then by Theorem 2.5, the graph $\mathfrak{L}\left(\Gamma_{2}(L)\right)$ is planar, which is not projective. Also without loss of generality we may assume that $\left|S_{12}\right|,\left|S_{13}\right| \in\{3,4\}$. Then one can easily check that the graph $\mathfrak{L}\left(\Gamma_{2}(L)\right)$ contains a copy of $A_{1}$, one of the listed graphs in [6], which is not projective. In addition, if we assume that $S_{12}, S_{13}$ or $S_{23}$ has at least five elements, then the graph $\mathfrak{L}\left(\Gamma_{2}(L)\right)$ contains a subgraph isomorphic to $K_{7}$, which is not projective. Therefore, for the projectivity of $\mathfrak{L}\left(\Gamma_{2}(L)\right)$, it is necessary that there exist unique $i$ and $j$, with $1 \leq i, j \leq 3$, such that $3 \leq\left|S_{i j}\right| \leq 4$ and $\left|S_{k k^{\prime}}\right| \leq 2$, for $k \in\{i, j\}$ and $\left\{k^{\prime}\right\}=\{1,2,3\} \backslash\{i, j\}$.

Case 2. $\left|\bigcup_{j=1}^{3} S_{j}\right|=4$. In this case, if $\left|S_{i j}\right| \leq 1$, for all $i, j \in\{1,2,3\}$, then, by Theorem 2.5, the graph $\mathfrak{L}\left(\Gamma_{2}(L)\right)$ is planar, which is not projective. Now, suppose that there exists unique $S_{i}$, with $1 \leq i \leq 3$, say $S_{1}$, such that $\left|S_{1}\right|=2$. If $\left|S_{23}\right| \geq 2$, then $\Gamma_{2}(L)$ contains a copy of $K_{2,4}$. Clearly, $\bar{\gamma}\left(\mathfrak{L}\left(\Gamma_{2}(L)\right)\right) \geq \bar{\gamma}\left(\mathfrak{L}\left(K_{2,4}\right)\right)$, and we have $\bar{\gamma}\left(\mathfrak{L}\left(K_{2,4}\right)\right)=2$. It implies that the graph $\mathfrak{L}\left(\Gamma_{2}(L)\right)$ is not projective. Also if $\left|S_{12}\right|=2$ and $\left|S_{23}\right|=1$, then the contraction of $\mathfrak{L}\left(\Gamma_{2}(L)\right)$ contains $B_{1}$, one of the listed graphs in [6], which is not projective. Now, we may assume that $S_{23}=\varnothing$. If $S_{12}$ or $S_{13}$ has at least four elements, then the graph $\mathfrak{L}\left(\Gamma_{2}(L)\right)$ contains a subgraph isomorphic to $K_{7}$, which is not projective. Also if $\left|S_{12}\right|=\left|S_{13}\right|=2$, then the graph $\mathfrak{L}\left(\Gamma_{2}(L)\right)$ contains a copy of $A_{1}$, one of the listed graphs in [6], which is not projective. Therefore, for the projectivity of $\mathfrak{L}\left(\Gamma_{2}(L)\right)$, it is necessary that $2 \leq\left|S_{i j}\right| \leq 3,\left|S_{i k}\right| \leq 1$ and $S_{j k}=\varnothing$, for $\{j, k\}=\{1,2,3\} \backslash\{i\}$, when $\left|S_{i}\right|=2$.

Case 3. $\left|\bigcup_{j=1}^{3} S_{j}\right|=5$. Suppose that $\left|S_{1}\right|=3$. If $S_{12}$ or $S_{13}$ has at least one element, then the graph $\mathfrak{L}\left(\Gamma_{2}(L)\right)$ contains a copy of $D_{17}$, one of the listed graphs in [6], which is not projective. Also if $S_{23}$ has at least one element, then the contraction of $\mathfrak{L}\left(\Gamma_{2}(L)\right)$ contains a copy of $E_{20}$, one of the listed graphs in [6], which is not a projective graph. Therefore, for the projectivity of $\mathfrak{L}\left(\Gamma_{2}(L)\right)$, it is necessary that $S_{12}=S_{13}=S_{23}=\varnothing$, when $\left|S_{1}\right|=3$. On the other hand, suppose that there exists unique $S_{i}$, with $1 \leq i \leq 3$, say $S_{1}$, such that $\left|S_{1}\right|=1$. If $S_{12}$ or $S_{13}$ has at least one element, then the contraction of $\mathfrak{L}\left(\Gamma_{2}(L)\right)$ contains a copy of $E_{20}$, 
one of the listed graphs in [6], which is not a projective graph. Also if $\left|S_{23}\right| \geq 2$, then the contraction of $\mathfrak{L}\left(\Gamma_{2}(L)\right)$ contains a copy of $D_{17}$, one of the listed graphs in [6], which is not a projective graph. Therefore, for the projectivity of $\mathfrak{L}\left(\Gamma_{2}(L)\right)$, it is necessary that $S_{12}=S_{13}=\varnothing$ and $\left|S_{23}\right| \leq 1$, when $\left|S_{1}\right|=1$.

Conversely, if one of the statements (i), (ii) or (iii) holds, then we show that $\mathfrak{L}\left(\Gamma_{2}(L)\right)$ is a projective graph.

First suppose that $\left|\bigcup_{j=1}^{3} S_{j}\right|=3$. If $\left|S_{12}\right|=\left|S_{13}\right|=2$ and $\left|S_{23}\right|=4$, then the graph $\Gamma_{2}(L)$ is pictured in Figure 10, which is planar and the graph $\mathfrak{L}\left(\Gamma_{2}(L)\right)$ is pictured in Figure 11, which is projective. We have $v_{1} \in S_{1}, v_{2} \in S_{2}, v_{3} \in S_{3}$, $v_{4}, v_{5} \in S_{12}, v_{6}, v_{7} \in S_{13}$ and $v_{8}, v_{9}, v_{10}, v_{11} \in S_{23}$.

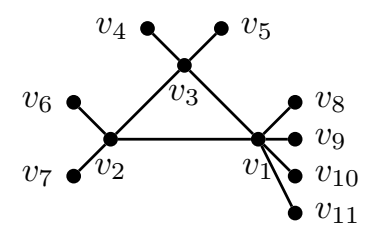

Figure 10

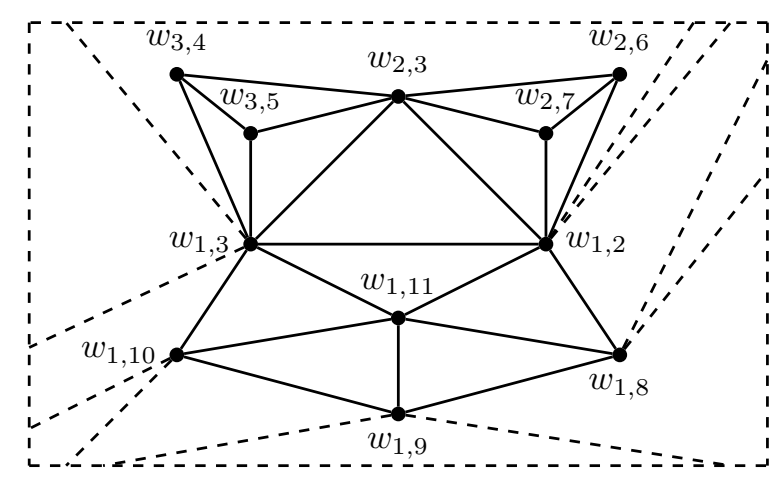

Figure 11

Now, suppose that $\left|\bigcup_{j=1}^{3} S_{j}\right|=4$ and $\left|S_{1}\right|=2$. If $\left|S_{12}\right|=1,\left|S_{13}\right|=3$ and $S_{23}=\varnothing$, then the graph $\Gamma_{2}(L)$ with vertices $v_{1}, v_{2} \in S_{1}, v_{3} \in S_{2}, v_{4} \in S_{3}, v_{5} \in S_{12}$ and $v_{6}, v_{7}, v_{8} \in S_{13}$ is planar and the graph $\mathfrak{L}\left(\Gamma_{2}(L)\right)$ is projective (see Figure 12).

Finally, suppose that $\left|\bigcup_{j=1}^{3} S_{j}\right|=5$ and consider the following cases.

Case 1. There exists unique $S_{i}$, with $1 \leq i \leq 3$, say $S_{1}$, such that $\left|S_{1}\right|=3$, and also $S_{12}=S_{13}=S_{23}=\varnothing$. Then the graph $\Gamma_{2}(L)$ with vertices $v_{1}, v_{2}, v_{3} \in S_{1}$, 


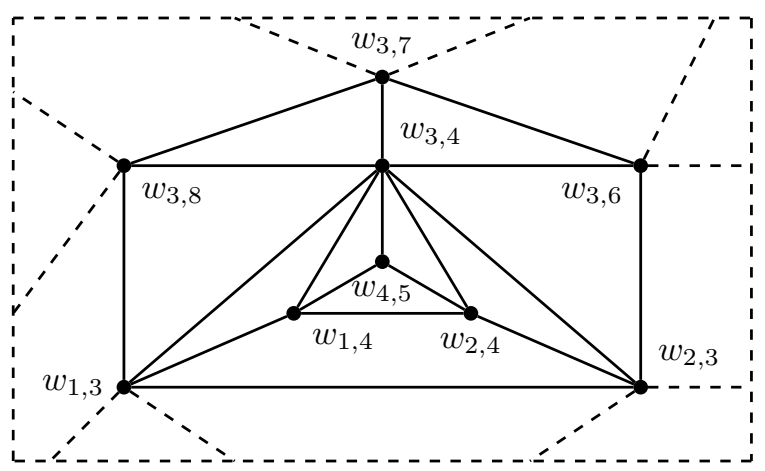

FiguRE 12

$v_{4} \in S_{2}$ and $v_{5} \in S_{3}$ is planar. As observed, in Figure 13, the graph $\mathfrak{L}\left(\Gamma_{2}(L)\right)$ is projective.

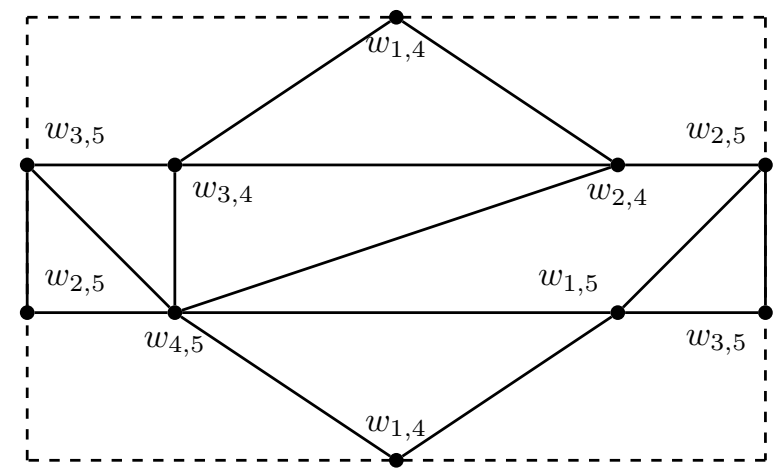

FiguRE 13

Case 2. There exists unique $S_{i}$, with $1 \leq i \leq 3$, say $S_{1}$, such that $\left|S_{1}\right|=1$, also $S_{12}=S_{13}=\varnothing$ and $\left|S_{23}\right|=1$. Then the graph $\Gamma_{2}(L)$ with vertices $v_{1} \in S_{1}$, $v_{2}, v_{3} \in S_{2}, v_{4}, v_{5} \in S_{3}$ and $v_{6} \in S_{23}$ is planar, and so $\mathfrak{L}\left(\Gamma_{2}(L)\right)$ is pictured in Figure 14, is a projective graph.

In the following, we assume that $|C(L)|=4$. Firstly, consider that $\left|\bigcup_{j=1}^{4} S_{j}\right|=5$ and $\left|S_{1}\right|=2$. Then $\mathfrak{L}\left(\Gamma_{2}(L)\right)$ contains a subgraph isomorphic to $E_{20}$, one of the listed graphs in [6], which is not a projective graph. Therefore we have the following lemma.

Lemma 3.5. If $\mathfrak{L}\left(\Gamma_{2}(L)\right)$ is projective, then $\left|\bigcup_{j=1}^{4} S_{j}\right|=4$.

In the sequel, suppose that $\left|\bigcup_{j=1}^{4} S_{j}\right|=4$. We have the following situations. 


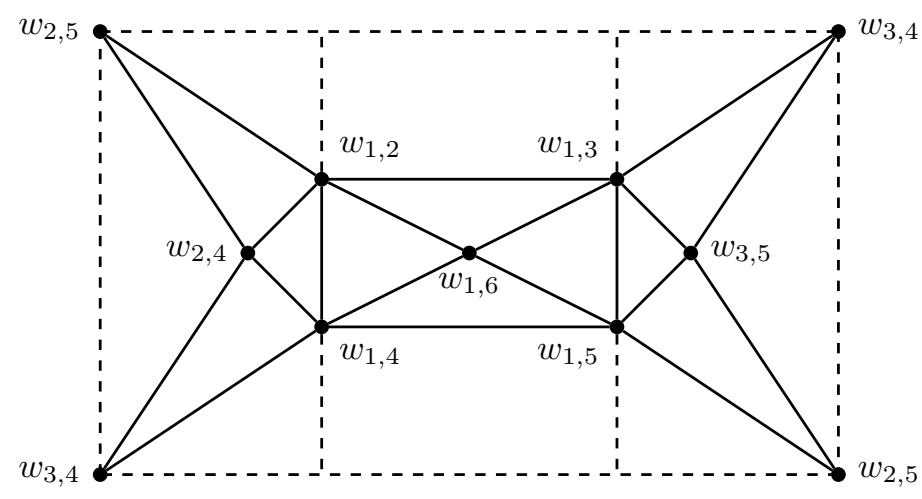

FIGURE 14

(i) There exist $i, j$, with $1 \leq i, j \leq 4$, such that $\left|S_{i j}\right| \geq 2$. Then $\mathfrak{L}\left(\Gamma_{2}(L)\right)$ contains a copy of $A_{1}$, one of the listed graphs in [6], which is not a projective graph.

(ii) There exist $i \neq i^{\prime}, j \neq j^{\prime}$, with $1 \leq i, i^{\prime}, j, j^{\prime} \leq 4$, such that $\left|S_{i j}\right|=\left|S_{i^{\prime} j^{\prime}}\right|=1$. Then the contraction $\mathfrak{L}\left(\Gamma_{2}(L)\right)$ contains a copy of $D_{17}$, one of the listed graphs in [6], which is not a projective graph.

(iii) There exist $i \neq i^{\prime}, j$, with $1 \leq i, i^{\prime}, j \leq 4$, such that $\left|S_{i j}\right|=\left|S_{i^{\prime} j}\right|=1$. Then $\mathfrak{L}\left(\Gamma_{2}(L)\right)$ contains a copy of $D_{17}$, one of the listed graphs in [6], which is not a projective graph.

(iv) For all $1 \leq i, j, k \leq 4,\left|S_{i j k}\right| \leq 1$ and $S_{i j}=\varnothing$. Then, by Theorem 2.7, the graph $\mathfrak{L}\left(\Gamma_{2}(L)\right)$ is planar, which is not projective.

(v) There exist $i, j, k$, with $1 \leq i, j, k \leq 4$, such that $\left|S_{i j k}\right| \geq 4$. Then $\mathfrak{L}\left(\Gamma_{2}(L)\right)$ contains a copy of $K_{7}$, which is not projective.

(vi) There exist unique $i \neq i^{\prime}, j, k$, with $1 \leq i, i^{\prime}, j, k \leq 4$, such that $2 \leq\left|S_{i j k}\right| \leq 3$ and $\left|S_{i^{\prime} i j}\right|=\left|S_{i^{\prime} i k}\right|=\left|S_{i^{\prime} j k}\right|=1$. Then the graph $\Gamma_{2}(L)$, with vertices $v_{1} \in S_{1}, v_{2} \in S_{2}, v_{3} \in S_{3}, v_{4} \in S_{4}, v_{5}, v_{6}, v_{7} \in S_{123}, v_{8} \in S_{124}, v_{9} \in S_{134}$ and $v_{10} \in S_{234}$ is planar. Therefore the graph $\mathfrak{L}\left(\Gamma_{2}(L)\right)$, which is pictured in Figure 15, is projective.

(vii) There exist $i \neq i^{\prime}, j, k$, with $1 \leq i, i^{\prime}, j, k \leq 4$, such that $\left|S_{i j k}\right|=\left|S_{i^{\prime} j k}\right|=2$. Then $\mathfrak{L}\left(\Gamma_{2}(L)\right)$ contains a copy of $A_{1}$, one of the listed graphs in [6], which is not a projective graph.

(viii) There exist $i, j \neq j^{\prime}, k \neq k^{\prime}$, with $1 \leq i, j, j^{\prime}, k, k^{\prime} \leq 4,\left|S_{i j}\right|=1$ and $\left|S_{i j^{\prime} k^{\prime}}\right|=$ 2. Then the contraction of $\mathfrak{L}\left(\Gamma_{2}(L)\right)$ contains a copy of $B_{1}$, one of the listed graphs in [6], which is not a projective graph. 


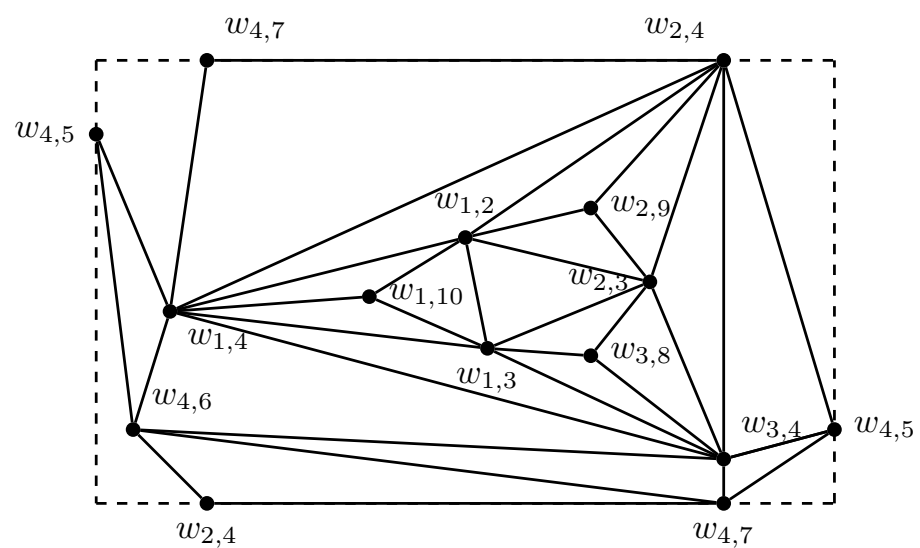

Figure 15

(ix) There exist $i, j, k$, with $1 \leq i, j, k \leq 4,\left|S_{i j}\right|=\left|S_{i j k}\right|=1$. Then $\mathfrak{L}\left(\Gamma_{2}(L)\right)$ contains a copy of $E_{19}$, one of the listed graphs in [6], which is not a projective graph.

(x) There exist unique $i, i^{\prime}, j, j^{\prime}$, with $\left\{i^{\prime}, j^{\prime}\right\}=\{1,2,3,4\} \backslash\{i, j\}$, such that $\left|S_{i j}\right|=$ $\left|S_{i i^{\prime} j^{\prime}}\right|=\left|S_{j i^{\prime} j^{\prime}}\right|=1$. Then the graph $\Gamma_{2}(L)$, with vertices $v_{1} \in S_{1}, v_{2} \in S_{2}$, $v_{3} \in S_{3}, v_{4} \in S_{4}, v_{5} \in S_{12}, v_{6} \in S_{134}$ and $v_{7} \in S_{234}$ is planar. Therefore the graph $\mathfrak{L}\left(\Gamma_{2}(L)\right)$, which is pictured in Figure 16, is projective.

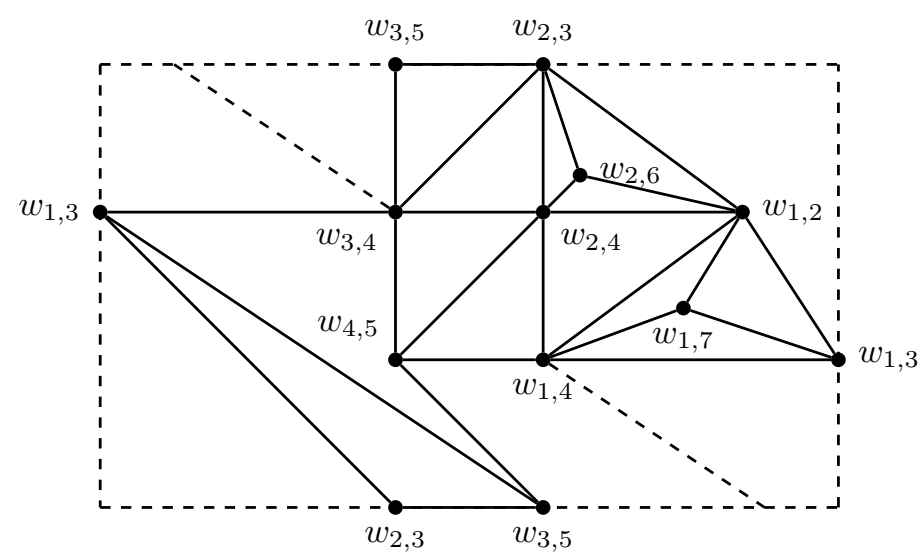

FiguRE 16

As a consequence of the above discussion and Lemma 3.5, we state the necessary and sufficient conditions for the projectivity of $\mathfrak{L}\left(\Gamma_{2}(L)\right)$, when $|C(L)|=4$. 
Theorem 3.6. Suppose that $|C(L)|=4$. Then $\mathfrak{L}\left(\Gamma_{2}(L)\right)$ is a projective graph if and only if $\left|\bigcup_{j=1}^{4} S_{j}\right|=4$ and one of the following conditions holds:

(i) There exist unique $i \neq i^{\prime}, j, k$, with $1 \leq i, i^{\prime}, j, k \leq 4$, such that $2 \leq\left|S_{i j k}\right| \leq 3$ and $\left|S_{i^{\prime} i j}\right|=\left|S_{i^{\prime} i k}\right|=\left|S_{i^{\prime} j k}\right|=1$.

(ii) There exist unique $i, i^{\prime}, j, j^{\prime}$, with $\left\{i^{\prime}, j^{\prime}\right\}=\{1,2,3,4\} \backslash\{i, j\}$, such that $\left|S_{i j}\right|=$ $\left|S_{i i^{\prime} j^{\prime}}\right|=\left|S_{j i^{\prime} j^{\prime}}\right|=1$.

Remark 3.7. Lattices have some connections to the family of group like structures. Because meet $(\wedge)$ and join $(\vee)$ both are commutative and associative, a lattice can be viewed as consisting of two commutative semigroups having the same domain. For a finite lattice, these semigroups are in fact commutative monoids. The concept of the comaximal graph was first defined for commutative rings and the semigroup properties of the two operations of rings were used. Hence if we have a semigroup with two operations, then the comaximal graph can be defined.

Acknowledgment. The authors are deeply grateful to the referee for careful reading of the manuscript and helpful suggestions.

\section{References}

[1] M. Afkhami and K. Khashyarmanesh, The comaximal graph of a lattice, Bull. Malays. Math. Sci. Soc., 37 (2014), 261-269.

[2] D. Archdeacon, A Kuratowski theorem for the projective plane, J. Graph Theory, 5 (1981), 243-246.

[3] J. A. Bondy and U. S. R. Murty, Graph Theory with Applications, American Elsevier, New York, 1976.

[4] A. Bouchet, Orientable and nonorientable genus of the complete bipartite graph, J. Combin. Theory Ser. B, 24 (1978), 24-33.

[5] H. J. Chiang-Hsieh, P. F. Lee and H. J. Wang, The embedding of line graphs associated to the zero-divisor graphs of commutative rings, Israel J. Math., 180 (2010), 193-222.

[6] H. Glover, J. P. Huneke and C. S. Wang, 103 graphs that are irreducible for the projective plane, J. Combin. Theory Ser. B, 27 (1979), 332-370.

[7] C. D. Godsil and G. Royle, Algebraic Graph Theory, Springer-Verlag, New York, 2001.

[8] H. R. Maimani, M. Salimi, A. Sattari and S. Yassemi, Comaximal graph of commutative rings, J. Algebra, 319 (2008), 1801-1808. 
[9] W. Massey, Algebraic Topology: An Introduction, Harcourt, Brace \& World, New York, 1967.

[10] W. Myrvold and J. Roth, Simpler projective plane embedding, Ars Combin., 75 (2005), 135-155.

[11] J. B. Nation, Notes on Lattice Theory, Cambridge studies in advanced mathematics, Vol. 60, Cambridge University Press, Cambridge, 1998.

[12] G. Ringel, Map Color Theorem, Springer-Verlag, New York/Heidelberg, 1974.

[13] J. Sedlacek, Some properties of interchange graphs, Theory of graphs and its applications, (M. Fiedler, ed.,) Progue 1962; Reprinted, Academic Press, New York (1962), 145-150.

[14] P. K. Sharma and S. M. Bhatwadekar, A note on graphical representation of rings, J. Algebra, 176 (1995), 124-127.

[15] H. J. Wang, Graphs associated to co-maximal ideals of commutative rings, J. Algebra, 320 (2008), 2917-2933.

[16] H. J. Wang, Co-maximal graph of non-commutative rings, Linear Algebra Appl., 430 (2009), 633-641.

[17] H. Whitney, Congruent graphs and connectivity of graphs, Amer. J. Math., 54 (1932), 150-168.

\author{
Mojgan Afkhami \\ Department of Mathematics \\ University of Neyshabur \\ P.O.Box 91136-899, Neyshabur, Iran \\ e-mail: mojgan.afkhami@yahoo.com
}

Kazem Khashyarmanesh and Atossa Parsapour

Department of Pure Mathematics

International Campus of Ferdowsi University of Mashhad

P.O.Box 1159-91775, Mashhad, Iran

e-mails: khashyar@ipm.ir (K. Khashyarmanesh)

parsa216@gmail.com (A. Parsapour) 